\title{
MOUND: An Alternative to Milgrom's MOND
}

\author{
Patrick G. Tonin \\ Saint Martin de Londres, France \\ Email:pgtonin@gmail.com
}

How to cite this paper: Tonin, P.G. (2021) MOUND: An Alternative to Milgrom's MOND. International Journal of Astronomy and Astrophysics, 11, 87-94. https://doi.org/10.4236/ijaa.2021.111006

Received: January 15, 2021

Accepted: March 6, 2021

Published: March 9, 2021

Copyright $\odot 2021$ by author(s) and Scientific Research Publishing Inc. This work is licensed under the Creative Commons Attribution International License (CC BY 4.0).

http://creativecommons.org/licenses/by/4.0/

\begin{abstract}
We test and explore a Modified Universe Dynamics (MOUND) formula recently proposed by the author. We show that, similarly to Milgrom's Modified Newtonian Dynamics (MOND), it is successful in accounting for the mass discrepancy in spiral galaxies, and it predicts the Baryonic Tully-Fisher Relation (BTFR) and the Radial Acceleration Relation (RAR). Contrary to Milgrom's MOND, MOUND also explains the dynamics of galaxy clusters and does not rely on an empirical interpolating function or an ad hoc acceleration parameter.
\end{abstract}

\section{Keywords}

Cosmological Models, Dark Matter, Modified Newtonian Dynamics

\section{Introduction}

Modified Newtonian Dynamics (MOND) is a modification of Newtonian gravity first suggested by Milgrom [1] as an alternative to dark matter to account for the mass discrepancy in spiral galaxies. Although notably successful in explaining rotation curves and kinematics of galaxies [1] [2], it falls short on galaxy clusters because additional undetected matter is still required [3] [4] [5]. Furthermore, MOND's formulation remains empirical and lacks an underlying fundamental theory, hence it is not generally accepted by the scientific community. In this paper, we explore a novel Modified Universe Dynamics (MOUND) formula recently proposed by the author [6]. In the MOUND approach, the Universe gravitational acceleration $g_{u}=3.4365 \times 10^{-10} \mathrm{~m} \cdot \mathrm{s}^{-2}$ couples with Newtonian acceleration, and Newtonian dynamics are preserved. The consequences of this coupling become apparent on large scales, when Newtonian acceleration is of the same magnitude as the Universe gravitational acceleration. We show that MOUND explains not only the dynamics of galaxies but also those of galaxy groups and clusters. We show that the formula reverts to the Newtonian formula 
on high accelerations and to the Baryonic Tully Fisher Relation (BTFR) [7] [8] on low accelerations. Finally, we demonstrate that it also predicts the Radial Acceleration Relation (RAR) [9].

\section{The MOUND Formula}

In a paper entitled “The Quantum Bang Hypothesis: an Alternative to Dark Matter and Dark Energy" [6], the author proposed the following MOUND formula for the observed rotation velocity $v_{o b s}$ of a galaxy:

$$
v_{o b s}=\sqrt{\frac{G M}{r}+\sqrt{G M g_{u}}}
$$

where $G$ is the Newtonian gravitational constant, $M$ is the mass of the galaxy, $r$ is the distance from the center of the galaxy and $g_{u}$ is the Universe gravitational acceleration defined as:

$$
g_{u}=\frac{G M_{u}}{R_{h}^{2}}=3.4365 \times 10^{-10} \mathrm{~m} \cdot \mathrm{s}^{-2}
$$

where $M_{u}$ is the mass of the observable Universe and $R_{h}$ is the Hubble radius.

We note that, contrary to MOND, the acceleration parameter in MOUND is not empirical but derived from cosmological parameters.

\section{Fits to Galaxies Curves}

To test the MOUND formula, we use the velocity contributions from the Spitzer Photometry and Accurate Rotation Curves (SPARC) database [10] and compute the baryonic velocity contribution $V_{b a r}$ as follows:

$$
V_{b a r}=\sqrt{V_{\text {gas }}^{2} d^{2}+V_{\text {disk }}^{2} Y_{\text {disk }}+V_{b u l}^{2} Y_{b u l}}
$$

where $V_{\text {gas }}$ is the gas velocity contribution, $V_{\text {disk }}$ the disk velocity contribution, $V_{b u l}$ the bulge velocity contribution, $Y_{\text {disk }}$ and $Y_{b u l}$ are the mass-to-light ratios $\left(\mathrm{M}^{\star} / \mathrm{L}\right)$ of disk and bulge components respectively and $d$ is the distance ratio defined as:

$$
d=\frac{D_{\text {mound }}}{D_{a}}
$$

where $D_{\text {mound }}$ is the inferred distance from MOUND and $D_{a}$ is the adopted distance from the SPARC database.

Using the classic Newtonian formula $M=V^{2} R / G$, we compute the baryonic mass and use it to compute the MOUND velocity using the MOUND formula (Equation (1)). We then compare the computed rotation curve with the observed rotation curve traced using $V_{o b s}$ from the SPARC database.

We assume the $\mathrm{M}^{\star} / \mathrm{L}$ ratios to be constant for each given galaxy. To obtain the best fit, we adjust the disk $M^{\star} / \mathrm{L}$ ratio (also the bulge ratio, if present) and the distance ratio $d$. Mass-to-light ratios and the distance ratio are the only free parameters.

Figures 1-3 show three examples of MOUND fits to rotation curves. The radius 


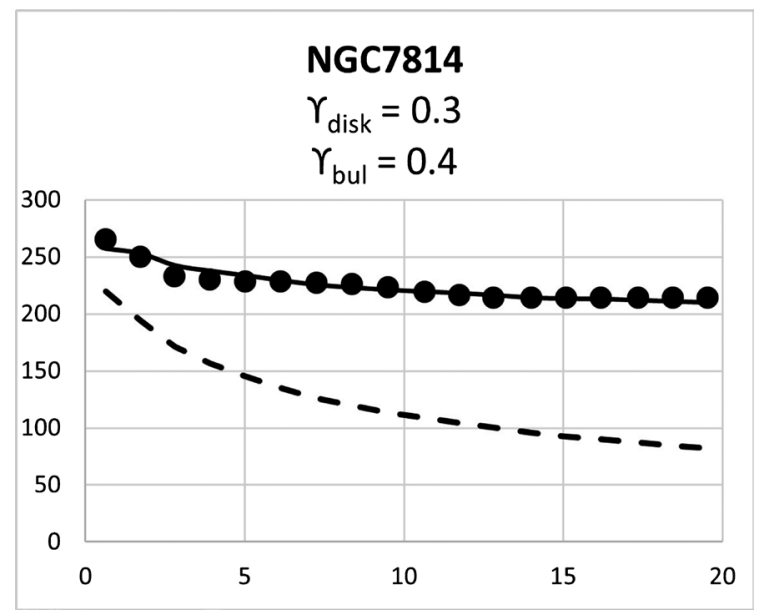

Figure 1. Bulge dominated spiral.

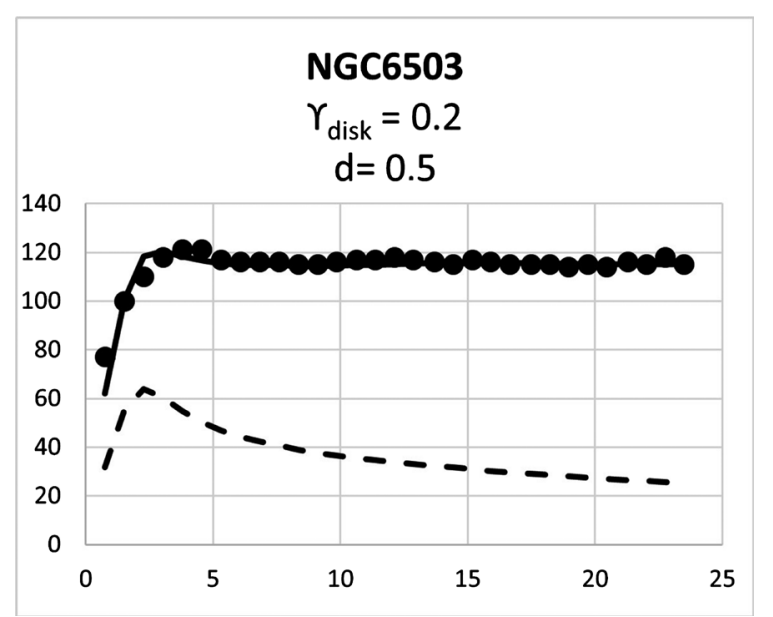

Figure 2. Disk dominated spiral.

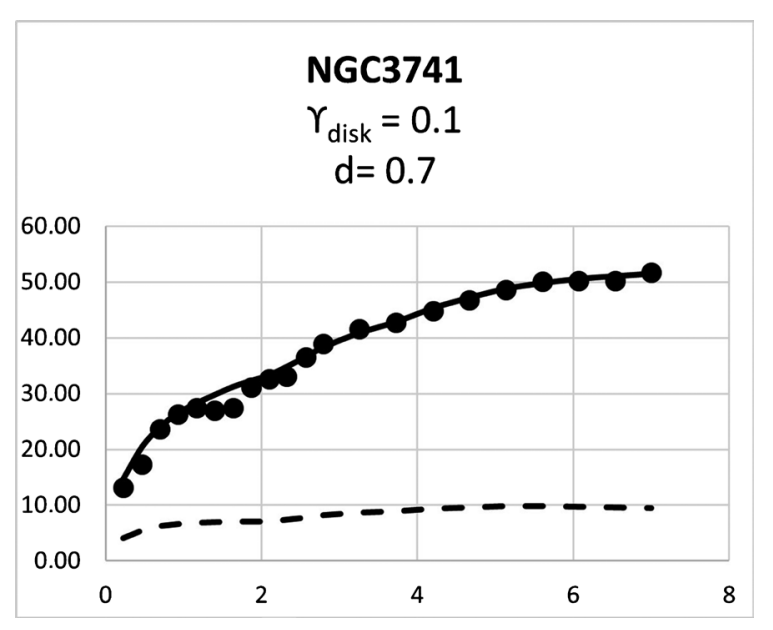

Figure 3. Gas dominated dwarf.

(horizontal axis) is given in $k p c$ and the rotation velocity (vertical axis) is given in $\mathrm{km} \cdot \mathrm{s}^{-1}$. The dots show the observed rotation curve $\left(V_{o b s}\right)$, the dashed line shows the baryonic Newtonian rotation curve $\left(V_{b a r}\right)$ and the solid line shows the 
MOUND predicted rotation curve $\left(V_{M O U N D}\right)$. For each galaxy, we show the inferred $M^{*} / \mathrm{L}$ ratio and the distance ratio $d$.

We applied the MOUND formula to a large sample of galaxies including small and large, bulge or disk dominated galaxies as well as gas dominated dwarfs, see Figures 1-3 and Figure 4. Considering the uncertainty relating to the observed
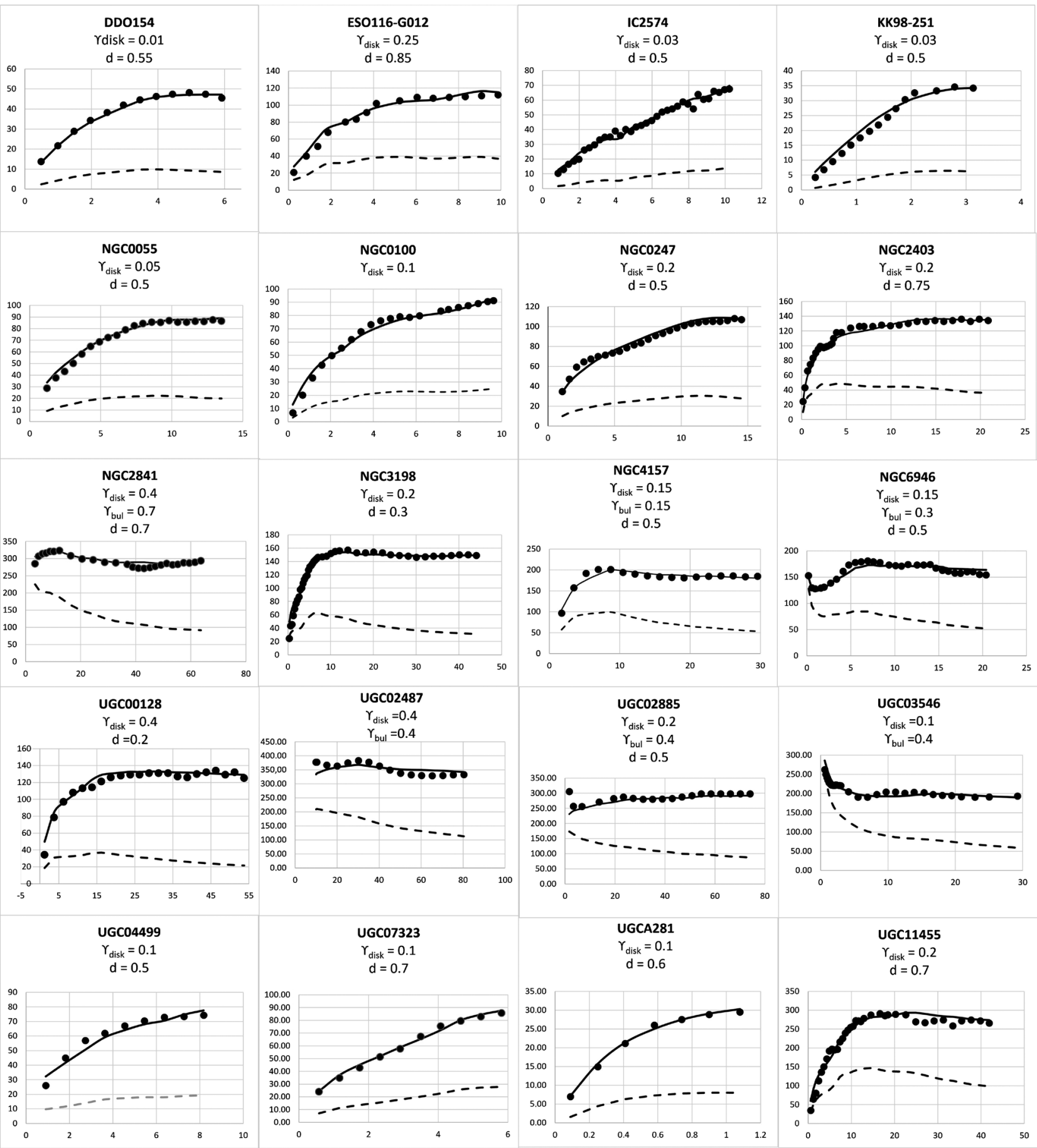

Figure 4. The radius (horizontal axis) is given in $k p c$ and the rotation velocity (vertical axis) is given in $\mathrm{km} \cdot \mathrm{s}^{-1}$. The dots show the observed rotation curve $\left(V_{o b s}\right)$, the dashed line shows the baryonic Newtonian rotation curve $\left(V_{b a r}\right)$ and the solid line shows the MOUND predicted rotation curve $\left(V_{M O U N D}\right)$. 
velocities, distances, inclinations and possible peculiar velocities of galaxies, the rotation curves obtained using the MOUND formula confirm that MOUND could account for the mass discrepancy in galaxies without the need to invoke dark matter.

\section{Galaxy Groups and Clusters}

Milgrom's MOND fails to account for the mass discrepancy in galaxy groups and clusters by a factor of two to three [3] [4] [5]. We show, in Figure 5, that this is not the case for MOUND.

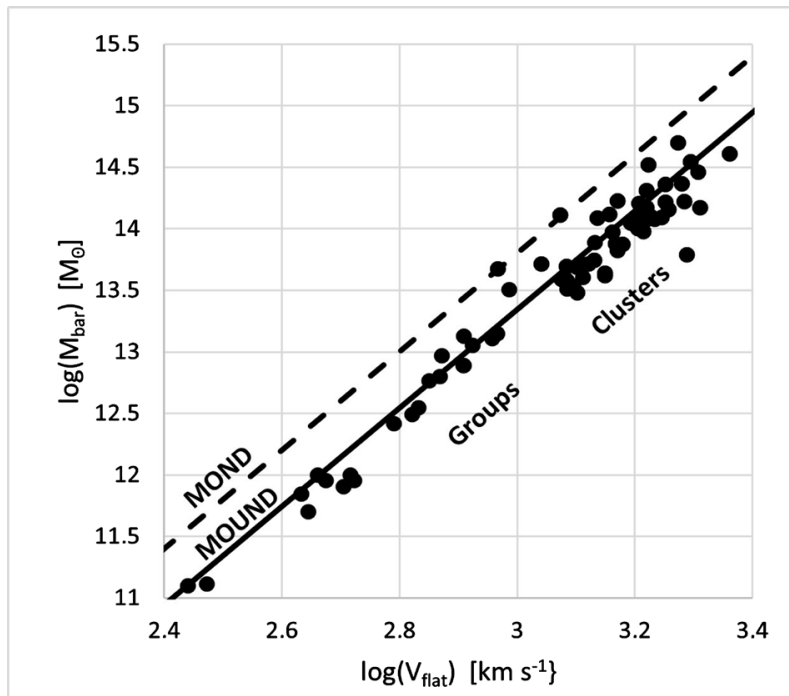

Figure 5. The baryonic mass as a function of circular velocity for galaxy groups and galaxy clusters (dots). The MOND prediction is shown as a dashed line and the MOUND prediction is shown as a solid line.

\section{The Classic Newtonian formula}

From equation (1), we deduce that:

if

$$
\frac{G M}{r} \gg \sqrt{G M g_{u}}
$$

then

$$
v_{\text {obs }}=\sqrt{\frac{G M}{r}}
$$

which is exactly the classic Newtonian equation for the rotation velocity of a galaxy.

The radial acceleration $(g)$ is defined as:

$$
g=\frac{G M}{r^{2}}
$$

Therefore, Equation (5) can be rewritten as:

$$
g \gg g_{u}
$$


We conclude that, for radial accelerations $(g)$ that are much larger than the Universe gravitational acceleration $\left(g_{u}\right)$, the MOUND formula reverts to the classic Newtonian formula.

\section{The Baryonic Tully-Fisher Relation (BTFR)}

The mass of a galaxy correlates with the amplitude of the flat rotation velocity. This is known as the Baryonic Tully-Fisher Relation [7] [8] expressed as follows:

$$
v_{\text {flat }}^{4}=G M g
$$

where $V_{f a t}$ is the flat rotation velocity, $G$ is the Newtonian gravitational constant, $M$ is the mass of the galaxy and $g$ is the radial acceleration.

From Equation (1), we deduce that:

if

$$
\frac{G M}{r} \ll \sqrt{G M g_{u}}
$$

Then

$$
v_{o b s}^{4}=G M g_{u}
$$

which is exactly the Baryonic Tully-Fisher relation.

Equation (10) can be rewritten as:

$$
g \ll g_{u}
$$

We conclude that, for radial accelerations $(g)$ that are much smaller than the Universe gravitational acceleration $\left(g_{u}\right)$, the MOUND formula reverts to the Baryonic Tully-Fisher relation.

We note that, contrary to Milgrom's MOND, MOUND does not require an empirical interpolating function to transition from the high acceleration regime (Newtonian regime) to the low acceleration regime (MOND regime).

\section{The Radial Acceleration Relation (RAR)}

The radial acceleration of rotationally supported galaxies correlates with the acceleration predicted by the observed distribution of baryons. This is known as the Radial Acceleration Relation (RAR) [9] [11].

The observed radial acceleration $\left(g_{o b s}\right)$ is defined as:

$$
g_{o b s}=\frac{v_{o b s}^{2}}{r}
$$

where $V_{o b s}$ is the observed rotation speed and $r$ the distance from the galaxy's center.

The baryonic acceleration $\left(g_{b a r}\right)$ is defined as:

$$
g_{b a r}=\frac{G M}{r^{2}}
$$

Substituting the above in Equation (1), we obtain:

$$
g_{\text {obs }}=g_{b a r}+\sqrt{g_{b a r} g_{u}}
$$




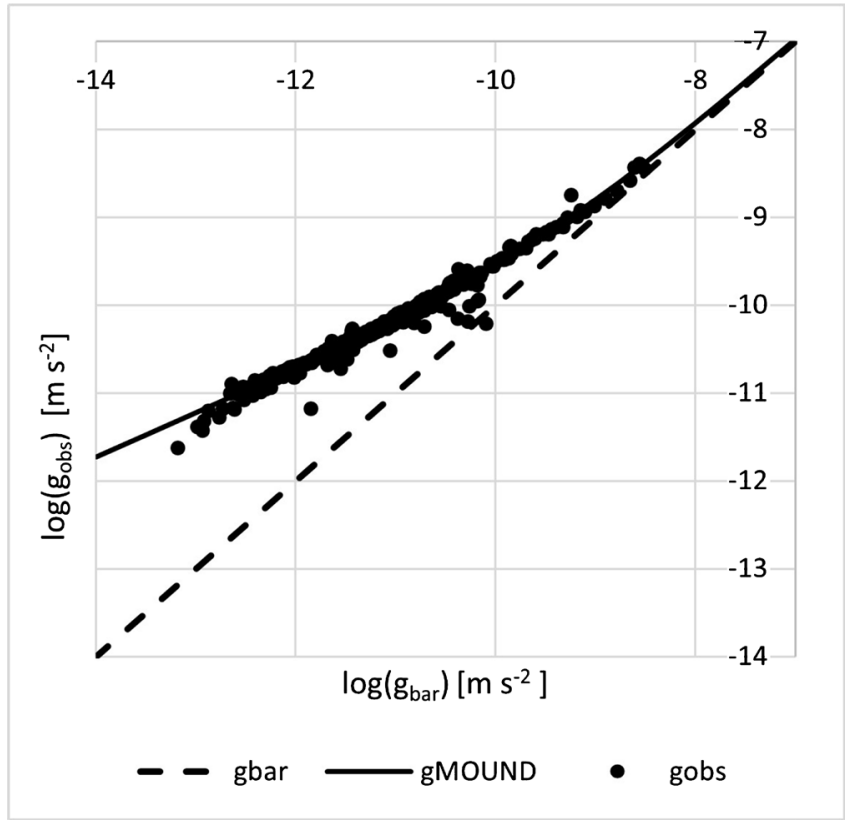

Figure 6. Observed radial acceleration versus the predicted baryonic acceleration. 516 data points from the 23 galaxies featured in this paper.

Figure 6 shows the observed radial acceleration (dots) versus the predicted baryonic acceleration (dashed line) and the MOUND predicted curve (solid line).

It confirms that the MOUND formula predicts, through a precise equation, that the observed radial acceleration is correlated to the baryonic acceleration as reported by McGaugh et al. [9]

\section{Conclusion}

In this paper, we have tested and explored a novel MOUND (Modified Universe Dynamics) formula recently proposed by the author. We have shown that it provides an alternative to Milgrom's MOND without the main limitations of MOND, namely its empirical interpolating function and its inability to fully account for the mass discrepancy in galaxy clusters. MOUND and its underlying theory [6] could potentially be a viable alternative to dark matter and form the basis of a much simpler cosmological model without any ad hoc parameters.

\section{Conflicts of interest}

The author declares no conflicts of interest regarding the publication of this paper.

\section{References}

[1] Milgrom, M. (1983) A Modification of the Newtonian Dynamics as a Possible Alternative to the Hidden Mass Hypothesis. The Astrophysical Journal, 270, 365-370. https://doi.org/10.1086/161130

[2] Sanders, R.H. andMcGaugh, S.S. (2002) Modified Newtonian Dynamics as an Alternative to Dark Matter. Annual Review of Astronomy and Astrophysics, 40, 
263-317. https://doi.org/10.1146/annurev.astro.40.060401.093923

[3] Pointecouteau, E. and Silk, J. (2005) New Constraints on Modified Newtonian Dynamics from Galaxy Clusters. Monthly Notices of the Royal Astronomical Society, 364, 654-658. https://doi.org/10.1111/j.1365-2966.2005.09590.x

[4] Sanders, R.H. (2003) Clusters of Galaxies with Modified Newtonian Dynamics. Monthly Notices of the Royal Astronomical Society, 342, 901-908. https://doi.org/10.1046/j.1365-8711.2003.06596.x

[5] The, L.S. and White, S.D.M. (1988) Modified Newtonian Dynamics and the Coma Cluster. Astronomical Journal, 95, 1642. https://doi.org/10.1086/114760

[6] Tonin, P.G. (2020) The Quantum Bang Hypothesis: An Alternative to Dark Matter and Dark Energy. Journal of High Energy Physics, Gravitation and Cosmology, 6, 753-764. https://doi.org/10.4236/jhepgc.2020.64050

[7] Tully, R.B. and Fisher, J.R. (1977) A New Method of Determining Distances to Galaxies. Astronomy and Astrophysics, 54, 661.

[8] McGaugh, S.S., Schombert, J.M., Bothun, G.D. and de Blok, W.J.G. (2000) The Baryonic Tully-Fisher Relation. The Astrophysical Journal, 533, L99. https://doi.org/10.1086/312628

[9] McGaugh, S.S., Lelli, F. and Schombert, J.M. (2016) The Radial Acceleration Relation in Rotationally Supported Galaxies. Physical Review Letters, 117, 201101. https://doi.org/10.1103/PhysRevLett.117.201101

[10] Lelli, F., McGaugh, S.S. andSchombert, J.M. (2016) SPARC: Mass Models for 175 Disk Galaxies with Spitzer Photometry and Accurate Rotation Curves. The Astronomical Journal, 152, 157. https://doi.org/10.3847/0004-6256/152/6/157

[11] Lelli, F., McGaugh, S.S., Schombert, J.M. and Pawlowski, M.S. (2017) One Law to Rule Them All: The Radial Acceleration Relation of Galaxies. The Astrophysical Journal, 836, 152. https://doi.org/10.3847/1538-4357/836/2/152 